\title{
Analysis of genetic variants in myeloproliferative neoplasms using a 22-gene next-generation sequencing panel
}

\author{
Jaymi Tan ${ }^{1}$, Yock Ping Chow ${ }^{2}$, Norziha Zainul Abidin³ ${ }^{3}$ Kian Meng Chang ${ }^{4}$, Veena Selvaratnam \\ Nor Rafeah Tumian', Yang Ming Poh', Abhi Veerakumarasivam', Michael Arthur Laffan ${ }^{8,9}$ and \\ Chieh Lee Wong ${ }^{1,2,3,4,8,9^{*}}$
}

\begin{abstract}
Background: The Philadelphia (Ph)-negative myeloproliferative neoplasms (MPNs), namely essential thrombocythaemia (ET), polycythaemia vera (PV) and primary myelofibrosis (PMF), are a group of chronic clonal haematopoietic disorders that have the propensity to advance into bone marrow failure or acute myeloid leukaemia; often resulting in fatality. Although driver mutations have been identified in these MPNs, subtype-specific markers of the disease have yet to be discovered. Next-generation sequencing (NGS) technology can potentially improve the clinical management of MPNs by allowing for the simultaneous screening of many disease-associated genes.
\end{abstract}

Methods: The performance of a custom, in-house designed 22-gene NGS panel was technically validated using reference standards across two independent replicate runs. The panel was subsequently used to screen a total of 10 clinical MPN samples ( $E T n=3, P V n=3, P M F n=4$ ). The resulting NGS data was then analysed via a bioinformatics pipeline.

Results: The custom NGS panel had a detection limit of 1\% variant allele frequency (VAF). A total of 20 unique variants with VAFs above $5 \%$ ( 4 of which were putatively novel variants with potential biological significance) and one pathogenic variant with a VAF of between 1 and $5 \%$ were identified across all of the clinical MPN samples. All single nucleotide variants with $V A F s \geq 15 \%$ were confirmed via Sanger sequencing.

Conclusions: The high fidelity of the NGS analysis and the identification of known and novel variants in this study cohort support its potential clinical utility in the management of MPNs. However, further optimisation is needed to avoid false negatives in regions with low sequencing coverage, especially for the detection of driver mutations in MPL.

Keywords: Myeloproliferative neoplasm, Next-generation sequencing, Gene, Mutation, Variant, Bioinformatics, disease management

\footnotetext{
*Correspondence: wongchiehlee@hotmail.com; wongcl@sunmed.com.my

${ }^{4}$ Haematology Unit, Department of Medicine, Sunway Medical Centre,

Petaling Jaya, Selangor Darul Ehsan, Malaysia

Full list of author information is available at the end of the article
}

\begin{abstract}
Background
Myeloproliferative neoplasms (MPNs) are a group of chronic clonal haematopoietic disorders. The MPN subtypes include the Philadelphia (Ph) chromosome-positive chronic myeloid leukaemia, as well as the Ph-negative MPNs such as essential thrombocythaemia (ET) (characterised by an overproduction of platelets), polycythaemia vera (PV) (characterised by an overproduction of red
\end{abstract}


blood cells), and primary myelofibrosis (PMF) (characterised by progressive bone marrow fibrosis, and is further subdivided into an early, prefibrotic stage (pre-PMF) and a late, overt fibrotic stage (overt-PMF)). Among the three, ET is the most indolent, whereas PMF is associated with the highest symptom burden and worst prognosis. Common causes of morbidity and mortality include thromboembolic and/or haemorrhagic complications, as well as disease progression to myelofibrosis (MF) and/ or transformation to acute myeloid leukaemia (AML), all of which vary in frequencies between MPN subtypes [1]. To date, allogenic stem cell transplantation remains the only curative option for MPNs but it is often not considered due to age-related co-morbidities and high transplant-associated mortality rates. Hence, available treatment options such as phlebotomy, aspirin, hydroxyurea, anagrelide, pegylated interferons, and JAK inhibitors are primarily aimed at reducing the risk of disease complications, disease progression, and malignant transformation [2,3].

The discovery of driver mutations in the JAK2, CALR and $M P L$ genes contributed towards the improved accuracy of MPN diagnostics. The JAK2 gene encodes the JAK2 non-receptor tyrosine kinase associated with several receptors which are critical for normal myelopoiesis, including the erythropoietin, thrombopoietin, and granulocyte colony-stimulating factor receptors; the $M P L$ gene encodes the thrombopoietin receptor; while the $C A L R$ gene encodes the chaperone protein calreticulin. Driver mutations in these genes (JAK2 p.V617F or exon 12 mutations, $C A L R$ exon 9 insertions and deletions, and MPL W515 mutations) lead to the constitutive activation of associated receptors and the subsequent amplification of downstream signalling pathways, which result in the aberrant cell proliferation in MPN. However, these driver mutations are not MPN subtype-specific, and can also be found in other myeloid diseases such as myelodysplastic syndromes (MDS), myelodysplastic/myeloproliferative neoplasms (MDS/MPN) and AML [4, 5]. In addition, there are also MPN cases that are triple-negative (TN) for all the driver mutations.

The management of MPNs requires an integrated, multimodal approach that includes the evaluation of clinical features, peripheral blood smear, bone marrow morphology, immunophenotype, cytogenetics, as well as molecular genetics, as recommended in the 2016 World Health Organisation (WHO) classification guidelines [4]. As MPNs are chronic in nature, cases are often asymptomatic upon diagnosis and discovered incidentally through physical examination or abnormal blood counts [4]. Typically, the diagnostic procedure includes molecular tests for MPN driver mutations, as well as the morphological analysis of the peripheral blood smear and bone marrow aspirate/trephine biopsy; the findings of which are correlated with the results of the full blood count. However, morphological features such as the degree of bone marrow fibrosis used for disease diagnosis are subjective in nature and thus have high inter-observer variability [6-8]. Therapeutic decisions are then made based on the patient's mutational landscape, disease burden, as well as disease prognosis. Nevertheless, the significant genotypic and phenotypic heterogeneity that exist between MPN subtypes and other myeloid disorders remains a challenge towards effective management of MPNs.

Rapid advancements in gene sequencing technology in the last decade have led to the discovery of other MPN-associated genetic aberrations, such as mutations in ASXL1, EZH2, TET2, IDH1, IDH2, SRSF2 and $S F 3 B 1$, contributing towards improved disease prognostication. In a novel prognostic model developed by Grinfeld, et al. [9], patients with MPN were stratified into eight groups by combining genomic data with traditional laboratory and clinical findings. Compared to current prognostic models, the model was superior in performance and was able to better define disease outcomes, especially within the "intermediate-risk" categories of the current prognostic models [9]. However, more comprehensive genetic profiling of patients using next-generation sequencing (NGS) technology is required before such a model can be implemented in clinics worldwide.

In this study, we report on the validation of a custom NGS panel which targets $22 \mathrm{MPN}$-associated genes (ABL1, ASXL1, CALR, CBL, CEBPA, CSF3R, DNMT3A, EZH2, FLT3, IDH1, IDH2, JAK2, KIT, MPL, NPM1, PDGFRA, RUNX1, SF3B1, SRSF2, TET2, TP53, and $U 2 A F 1$ ) by using a set of reference standards and a small cohort of clinical MPN samples.

\section{Materials and methods \\ Ethics statement}

A multicentre study was conducted across three institutions in Malaysia, namely Sunway Medical Centre, Ampang Hospital, and Universiti Kebangsaan Malaysia Medical Centre. Ethics approval for this study was obtained from the Sunway Medical Centre Independent Research Ethics Committee (SREC 008/2018/ FR), Universiti Kebangsaan Malaysia Medical Centre Research Ethics Committee (UKM FPR.4/244/FF-2018420), Medical Research \& Ethics Committee Ministry of Health Malaysia (MREC 8KKM/NIHSEC/P-19-99), and Sunway University Research Ethics Committee (PGSUREC 2019/010). 


\section{Custom NGS panel}

An Ampliseq for Illumina custom NGS panel was designed using the Illumina Design Studio tool (Illumina, San Diego, USA) to target the hotspot exons of 22 genes known to be frequently mutated in MPNs, namely $A B L 1$, ASXL1, CALR, CBL, CEBPA, CSF3R, DNMT3A, EZH2, FLT3, IDH1, IDH2, JAK2, KIT, MPL, NPM1, PDGFRA, RUNX1, SF3B1, SRSF2, TET2, TP53, and U2AF1 (Additional file 1: Table S1 and Additional file 2: Table S2).

\section{Technical validation of custom NGS panel performance}

The custom NGS panel performance was validated using four reference standards through two identical but independent NGS runs (Additional file 3: Table S3). The reference standards used were: (1) Tru-Q0 (100\% wild-type) Reference Standard (Horizon, Cambridge, UK)-as a negative control; (2) Tru-Q1 (5\% Tier) Reference Standard (Horizon) -as a positive control for $5 \%$ mutant allele in FLT3 (p. $\triangle \mathrm{I} 836$ ), IDH1 (p.R132C), and JAK2 (p.V617F); 3) Tru-Q7 (1.3\% Tier) Reference Standard (Horizon) - as a positive control for $1.3 \%$ mutant allele in FLT3 (p.D835Y, p. $\triangle \mathrm{I} 836$ ), IDH1 (p.R132C/H), IDH2 (p.R140Q, p.R172K), JAK2 (p.V617F), KIT (p.D816V), and PDGFRA (p.D842V); and 4) Seraseq ${ }^{\mathrm{TM}}$ Myeloid Mutation DNA Mix (SeraCare, Milford, USA)-as a positive control for mutant allele in $A B L 1$ (p.T315I), $A S X L 1$ (p.E635fs*15, p.G646fs*12), CALR (p.L367fs*46), $C B L$ (p.R420Q, p.L380P), CEBPA (p.H24fs*84, p.K313_ V314insK), CSF3R (p.T618I), FLT3 (c.1759_1800dup, duplication of chr13:28,608,250-28,608,277, p.D835Y), IDH1 (p.R132C), JAK2 (p.V617F, p.N542_E543del), MPL (p.W515L), NPM1 (p.W288fs*12), SF3B1 (p.K700E, p.K666N), SRSF2 (p.P95_R102del), and U2AF1 (p.S34F) (Additional file 3: Table S3). The NGS assay performance was assessed based on the sensitivity, specificity, repeatability, concordance, and positive predictive values.

\section{Study cohort}

Ten patients who were clinically diagnosed with ET, PV or PMF according to the 2016 WHO classification guidelines [4] in the participating institutions were recruited for this study. All patients who agreed to participate in the study provided their written consent. Approximately $10 \mathrm{~mL}$ peripheral blood samples from each patient were collected in EDTA tubes and processed within $24 \mathrm{~h}$ to extract the DNA from the cells. Patient demographic and clinical data were recorded by using standardised forms.

\section{Genomic DNA extraction and quality control}

Genomic DNA was extracted from each blood sample using the Maxwell ${ }^{\circledR}$ RSC Whole Blood DNA Kit (Promega, Madison, USA) according to the manufacturer's guidelines. The purity $\left(\mathrm{A}_{260} / \mathrm{A}_{280}\right.$ and $\left.\mathrm{A}_{260} / \mathrm{A}_{230}\right)$ and concentration of extracted DNA were assessed using the Nanodrop ${ }^{\mathrm{TM}}$ (Thermo Fisher Scientific, Waltham, USA) spectrophotometer. DNA samples with satisfactory purity ratios of $1.80-2.00\left(\mathrm{~A}_{260} / \mathrm{A}_{280}\right)$ and $2.00-2.20$ $\left(\mathrm{A}_{260} / \mathrm{A}_{230}\right)$ were visualised using $1 \%$ agarose gel electrophoresis and stored for downstream experiments.

\section{Preparation of NGS libraries and sequencing}

The NGS libraries were prepared according to the AmpliSeq for Illumina On-Demand, Custom and Community Panels Reference Guide Protocol, DNA Panels Standard Workflow Procedure for Three Primer Pools, using reagents from the AmpliSeq ${ }^{\mathrm{TM}}$ Library PLUS for Illumina ${ }^{\circledR}$. The concentration of the input DNA was measured using the Qubit dsDNA BR Assay and the concentration of the constructed libraries was assessed using the Qubit dsDNA HS Assay on the Qubit 2.0 Fluorometer (Thermo Fisher Scientific) following the manufacturer's protocol. The quality of the constructed libraries was assessed using the DNA Hi Sens Lab Chip (PerkinElmer, Waltham, USA) according to the DNA High Sensitivity Assay User Guide for LabChip GX Touch/GXII Touch Standard Sample Workflow. Libraries were denatured and diluted according to the MiSeq System Denature and Dilute Libraries Guide Protocol A: Standard Normalization Method for MiSeq Reagent Kit v2, with a PhiX library used as a sequencing control. Pooled sequencing of 6 samples per run was conducted on an Illumina Miseq using the MiSeq Reagent Micro Kit v2 ( $2 \times 150$ base pair (bp), paired end) to a minimum coverage depth of $5000 \times$ according to the manufacturer's protocol.

\section{Quality control and bioinformatics analysis of NGS data}

Figure 1 shows a schematic representation of the steps involved in the quality control and bioinformatics analysis of the NGS data. The sequencing metrics were visualized using the Illumina Sequence Analysis Viewer software. The quality of the raw NGS data was assessed using the FastQC software on the Illumina BaseSpace ${ }^{\mathrm{TM}}$ Sequence Hub. The sequencing data was analysed using a combination of two sequence alignment and variant calling applications (apps) also on the Illumina BaseSpace $^{\text {TM }}$ Sequence Hub-the DNA Amplicon app and Pindel app, at a $1 \%$ somatic variant allele frequency (VAF) detection limit, aligned against the Genome Reference Consortium human genome build 37 (GRCh37). The DNA Amplicon app is designed to detect small variants, whereas the Pindel app is designed to detect larger structural variants such as large deletions (as large as $10 \mathrm{~kb}$ ), medium sized insertions, inversions and tandem duplications [10]. The DNA Amplicon app also generates a detailed report that summarises various 


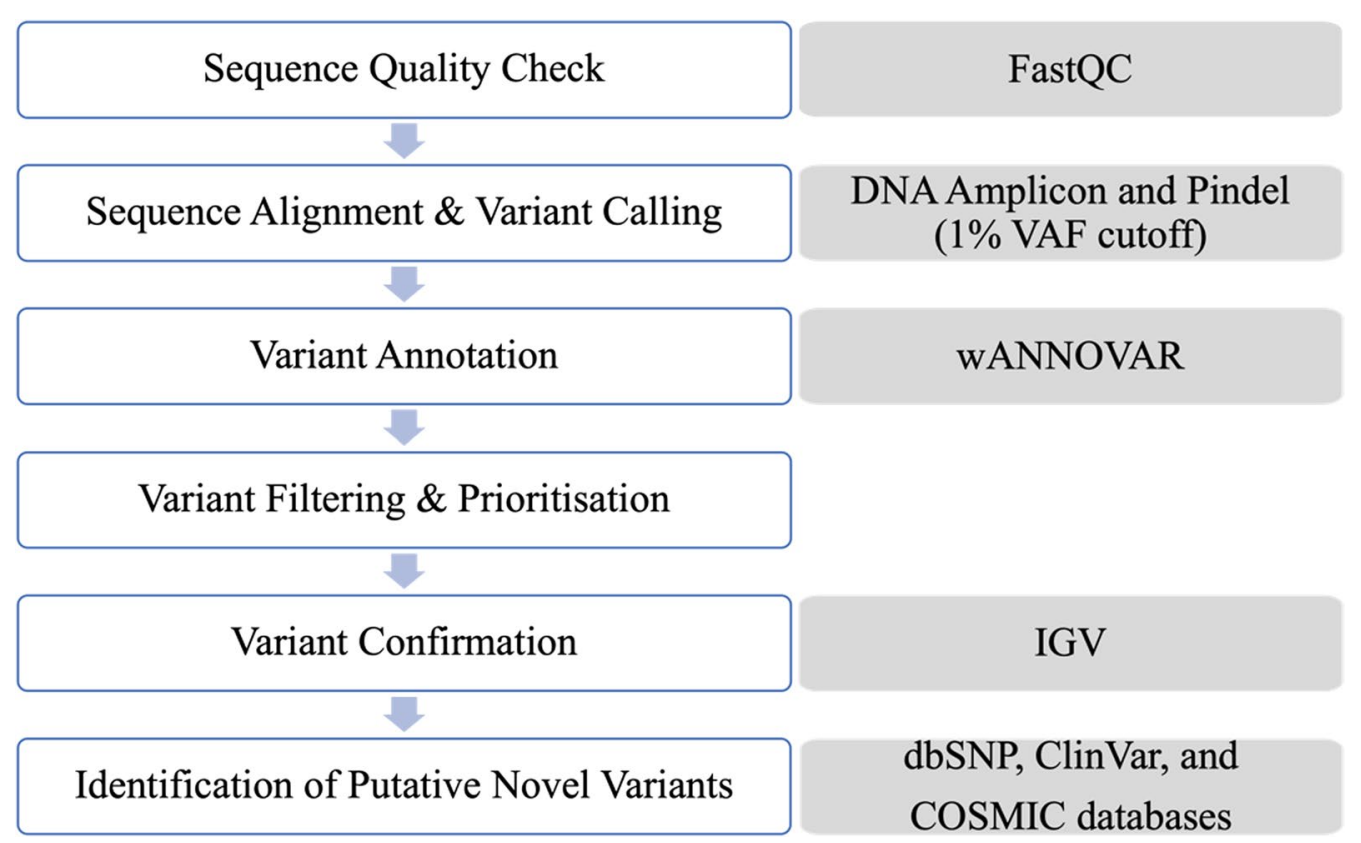

Fig. 1 Schematic representation of steps involved in the quality control and bioinformatics analysis of the NGS data. The variant filtering and prioritisation step is further illustrated in Fig. 3. Adapted from Dai et al., 2019 [11] and Zheng et al., 2018 [12]

information including sample read quality, amplicon data, base level statistics, and coverage by amplicon region. The called variants were then annotated using wANNOVAR (Wang Genomics Lab, Philadelphia, USA).

For the technical validation of the custom NGS panel, only variants that were present in the four reference standards were filtered-in and prioritized; whereas for the screening of the clinical MPN samples, all annotated variants were manually reviewed by filtering and prioritizing using the following criteria: (1) all variants except for exonic variants were excluded, (2) variants with minor allele frequencies (MAFs) of $\geq 1 \%$ (as reported in the 1000 Genomes Project, ExAC, ESP6500, and gnomAD databases) were excluded, and (3) potential sequencing errors (variants with VAF of $<5 \%$ and/or appear in majority of the samples) were excluded. In addition, variants with VAFs between 1 and $5 \%$ were inspected to check for any pathogenic/ likely pathogenic variants. Aligned read (.bam) files for all samples were then manually inspected to confirm the presence of the filtered-in and prioritized variants using the Integrative Genomics Viewer (IGV) software (Broad Institute, Cambridge, USA). In order to identify any putative novel variants, the variants were manually checked against dbSNP [13] as well as the ClinVar [14] and COSMIC [15] databases to determine if they had been previously reported as pathogenic.

\section{Variant confirmation via Sanger sequencing}

The Ensembl genome browser [16] and NCBI PrimerBLAST [17] were used to design PCR primers for the target variants (primer sequences are listed in Additional file 4: Table S4). Each PCR mixture contained 17 $\mu \mathrm{L}$ of nuclease free water, $25 \mu \mathrm{L}$ of the Amplitaq Gold ${ }^{\circledR}$ 360 Master Mix (Thermo Fisher Scientific), $2 \mu \mathrm{L} 360$ GC Enhancer (Thermo Fisher Scientific), $2 \mu \mathrm{L}$ each of $5 \mu \mathrm{M}$ forward and reverse primer, and $2 \mu \mathrm{L}$ of $50 \mathrm{ng} / \mu \mathrm{L}$ sample DNA. For the TET2 exon 7 p.D1314Mfs" 48 variant, the 360 GC Enhancer in the PCR mixture was found to reduce the PCR product yield, and was therefore replaced with nuclease free water. The PCR thermocycling conditions for all NGS detected variants were as follows: $95^{\circ} \mathrm{C}$ for $10 \mathrm{~min}, 35$ cycles at $95^{\circ} \mathrm{C}$ for $30 \mathrm{~s}, 55^{\circ} \mathrm{C}$ for $30 \mathrm{~s}$, and $72{ }^{\circ} \mathrm{C}$ for $1 \mathrm{~min}$, followed by a final extension at $72{ }^{\circ} \mathrm{C}$ for $7 \mathrm{~min}$. The PCR products were visualised using a $1 \%$ agarose gel electrophoresis to confirm successful amplification and then purified using Wizard ${ }^{\circledR}$ SV Gel and PCR Clean-Up System (Promega) by centrifugation according to the manufacturer's instructions but eluted in $15 \mathrm{~mL}$ of nuclease free water twice to obtain higher product concentration. The concentration and purity of the PCR products were assessed using the Nanodrop ${ }^{\mathrm{TM}}$ spectrophotometer as previously described. Samples with satisfactory concentration and purity ratios were sent for Sanger sequencing. Alignment of Sanger sequence (.seq) files were conducted via the ClustalW algorithm using 
the Jalview software [18] whereas the Sanger sequence chromatograms were analysed using the 4peaks software (Nucleobytes, Aalsmeer, Netherlands). The pathogenicity of the variants was assessed using ClinVar [14].

\section{Results}

\section{Technical validation of the custom NGS panel}

The custom NGS panel performance was evaluated using a set of reference standards in two identical but independent NGS runs. After library preparation, all samples were confirmed to be of sufficient quality and quantity. The majority of the constructed libraries were within the targeted size range of $400 \mathrm{bp}$ for sequencing using the custom NGS panel (Additional file 5: Fig. S1). Both NGS runs achieved cluster densities of 860 to $878 \mathrm{~K} / \mathrm{mm}^{2}$, $>93 \%$ of clusters passed the quality filter, and $>95 \%$ of the read bases with quality scores of above Q30, which were close to The Miseq System specifications of 865$965 \mathrm{~K} / \mathrm{mm}^{2}$ cluster density and $>80 \%$ of bases above Q30 (Additional file 6: Table S5). All samples achieved 99.5\% on-target aligned reads and minimum amplicon mean coverage depths of between $4589 x$ to $7944 x$ (Additional file 7: Table S6). Analysis of the coverage depth per amplicon region revealed that $98.6 \%$ of the targeted regions ( $n=216 / 219$ amplicons) had average coverage depths of $>1000 x$. Two amplicons had average coverage depths of <1000x, namely AMPL89337 (DNMT3A exon 17, chr2:25464411-25464625) and AMPL1156 (TP53 exon 4/exon 5, chr17:7578360-7578579), while AMPL117202 (MPL exon 10, chr1:43814902-43815103) had a coverage depth of below 100x (Additional file 8: Fig. S2).

Combined analysis of sequencing results with the DNA Amplicon and Pindel apps revealed that the former was able to detect all known variants in the reference standards except for large duplications in FLT3; whereas Pindel was able to detect all frameshift variants as well as large duplications in FLT3, but not SNVs (Fig. 2). Overall, the custom NGS panel has a sensitivity of $99.2 \%$, a specificity of $96.3 \%$, a positive predictive value of $97.7 \%$, an average intra-run concordance of 98.8\% [range 95.2-100\%], an average inter-run concordance of $99.0 \%$ [range 95.2$100 \%$, and a detection limit of $1 \%$ VAF (Fig. 2).

\section{Study cohort demographics and clinical data}

A total of $10 \mathrm{MPN}$ patients (ET $\mathrm{n}=3, \mathrm{PV} \mathrm{n}=3, \mathrm{PMF}$ $\mathrm{n}=4$ ) were recruited for this study (Sunway Medical Centre $(\mathrm{n}=7)$, Ampang Hospital $(\mathrm{n}=1)$ and Universiti Kebangsaan Malaysia Medical Centre $(n=2)$ ) (Table 1). All patients were diagnosed based on the latest WHO criteria [4]. The median age of diagnosis was 52 years (range: $30-79$ years). The majority of the patients were male $(n=7 / 10)$. Half of the study cohort was of Chinese ethnicity, 4 were Malay, and 1 was Dutch. Six patients presented with constitutional symptoms, and only 1 patient (Sample 09, overt-PMF) presented with hepatosplenomegaly. Half of the study cohort had a history of smoking. Seven patients were tested positive for the $J A K 2$ V617F driver mutation, and 3 were positive for $C A L R$ driver mutations. No patients tested positive for $M P L$ driver mutations.

\section{Identification of genetic variants in clinical MPN samples}

An initial total of 314 unique variants were detected across all 10 clinical MPN samples (Fig. 3). After filtering out intronic and UTR variants as well as all variants with MAFs of $\geq 1 \%, 115$ exonic variants remained. Subsequently, variants determined as sequencing errors were excluded. Ultimately, a total of 20 unique variants (which include known MPN driver mutations) with VAFs above $5 \%$ were identified across the 10 clinical MPN samples, and one variant with a VAF of $1.4 \%$ was found to be pathogenic as listed in the COSMIC database (COSM97191) (Table 2).

On average, the PMF samples appeared to harbour the highest number of variants, whereas the PV samples appeared to harbour the least number of variants (Fig. 4). Among the 20 unique variants with $\mathrm{VAF}>5 \%, 13$ were SNVs (synonymous SNV (sSNV) $\mathrm{n}=2$, nonsynonymous SNV (nSNV) $\mathrm{n}=11$ ) and 7 were indels (frameshift insertion (fs ins), $\mathrm{n}=2$; frameshift deletions (fs del), $\mathrm{n}=3$; stopgain, $\mathrm{n}=2$ ). Out of the 10 sequenced clinical samples, the $J A K 2$ V617F driver mutation was identified in 7 samples, while $C A L R$ driver mutation was identified in 3 samples. Aside from driver mutations, other variants were also detected, including an nSNV in CALR as well as variants in $A B L 1(\mathrm{n}=1), A S X L 1(\mathrm{n}=4), D N M T 3 A$ $(\mathrm{n}=1) ; \operatorname{RUNX1}(\mathrm{n}=1), \operatorname{SF} 3 B 1(\mathrm{n}=1), \operatorname{TET} 2(\mathrm{n}=6)$, and U2AF1 $(\mathrm{n}=2)$ (Fig. 4). All NGS-detected variants with allele frequencies of $\geq 15 \%$ were confirmed via Sanger sequencing (Table 2), except for the DNMT3A sSNV that was not confirmed due to nonspecific amplification (Additional file 9: Fig. S3).

The $A B L 1$ p.Asn350Ser point mutation identified in this study (Sample 07, pre-PMF) was reported in dbSNP (rs144448357). However, it is unknown whether it was previously identified in MPN due to the lack of ClinVar and COSMIC data (Table 2). The variant was identified in a patient diagnosed with pre-PMF at 50 years of age, with $J A K 2$ V617F mutation. The patient presented with increased platelet $\left(859 \times 10^{9} / \mathrm{L}\right)$ and white cell counts $\left(17.8 \times 10^{9} / \mathrm{L}\right)$, as well as constitutional symptoms (Table 1).

Four ASXL1 variants were identified in this study, of which, the ASXL1 p.Gly646Trpfs*10 (c.1927_1928insGGGGGGGGTGGCCCGGGTGGA GGTGGCGGCGGGGCCACCGATGAGGGGGGG 


\begin{tabular}{|c|c|c|c|c|c|c|c|c|c|c|c|c|c|}
\hline \multirow[b]{3}{*}{ Gene } & \multirow[b]{3}{*}{ Protein Change } & \multicolumn{6}{|c|}{ Run 1} & \multicolumn{6}{|c|}{ Run 2} \\
\hline & & \multirow[b]{2}{*}{$\begin{array}{l}\Xi \\
\Xi \\
己\end{array}$} & \multirow[b]{2}{*}{ 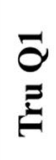 } & \multicolumn{2}{|c|}{ Tru Q7 } & \multicolumn{2}{|c|}{ Seraseq } & \multirow[b]{2}{*}{$\begin{array}{l}8 \\
\Xi \\
\end{array}$} & \multirow[b]{2}{*}{$\begin{array}{l}\bar{O} \\
\bar{D}\end{array}$} & \multicolumn{2}{|c|}{ Tru Q7 } & \multicolumn{2}{|c|}{ Seraseq } \\
\hline & & & & $\overrightarrow{\frac{2}{2}}$ & 记 & $\begin{array}{l}\vec{z} \\
\overline{2} \\
\simeq\end{array}$ & 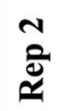 & & & $\overrightarrow{0}$ & 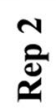 & 定 & ̃ै \\
\hline$A B L 1$ & p.T315I & & & & & $\mathrm{TP}$ & TP & & & & & TP & TP \\
\hline \multirow{2}{*}{$A S X L 1$} & p.E635fs*15 & & & & & TP & TP & & & & & TP & TP \\
\hline & p.G646fs*12 & FP & & & & $\mathrm{TP}$ & $\mathrm{TP}$ & FP & & & & $\mathrm{TP}$ & $\mathrm{TP}$ \\
\hline CALR & p.L367fs*46 & & & & & TP & $\mathrm{TP}$ & & & & & $\mathrm{TP}$ & TP \\
\hline \multirow[t]{2}{*}{$C B L$} & p.R420Q & & & & & $\mathrm{TP}$ & TP & & & & & $\mathrm{TP}$ & TP \\
\hline & p.L380P & & & & & $\mathrm{TP}$ & $\mathrm{TP}$ & & & & & TP & TP \\
\hline \multirow[t]{2}{*}{$C E B P A$} & p.H24fs*84 & & & & & TP & TP & & & & & $\mathrm{TP}$ & TP \\
\hline & p.K313_V314insK & & & & & $\mathrm{TP}$ & TP & & & & & TP & TP \\
\hline CSF3R & p.T618I & & & & & $\mathrm{TP}$ & $\mathrm{TP}$ & & & & & $\mathrm{TP}$ & $\mathrm{TP}$ \\
\hline \multirow[t]{4}{*}{ FLT3 } & c.1759_1800dup & & & & & TP & TP & & & & & TP & $\mathrm{TP}$ \\
\hline & dup chr13:28,608,250-28,608,277 & & & & & TP & TP & & & & & TP & TP \\
\hline & p.D835Y & & & $\mathrm{TP}$ & $\mathrm{TP}$ & TP & TP & & & TP & $\mathrm{TP}$ & TP & TP \\
\hline & p. $\Delta \mathrm{I} 836$ & & & TP & $\mathrm{TP}$ & & & & & $\mathrm{TP}$ & $\mathrm{TP}$ & & \\
\hline IDHI & p.R132C/H & & $\mathrm{TP}$ & $\mathrm{TP}$ & $\mathrm{TP}$ & TP & $\mathrm{TP}$ & & $\mathrm{TP}$ & $\mathrm{TP}$ & TP & TP & TP \\
\hline \multirow[t]{2}{*}{$\mathrm{IDH} 2$} & p.R140Q & & & TP & $\mathrm{TP}$ & & & & & $\mathrm{TP}$ & $\mathrm{TP}$ & & \\
\hline & p.R172K & & & TP & TP & & & & & TP & TP & & \\
\hline \multirow[t]{2}{*}{$J A K 2$} & p.V617F & & $\mathrm{TP}$ & $\mathrm{TP}$ & $\mathrm{TP}$ & TP & $\mathrm{TP}$ & & $\mathrm{TP}$ & $\mathrm{TP}$ & $\mathrm{TP}$ & $\mathrm{TP}$ & TP \\
\hline & p.N542_E543del & & & & & TP & TP & & & & & TP & TP \\
\hline KIT & p.D816V & & & TP & $\mathrm{TP}$ & & & & & $\mathrm{TP}$ & $\mathrm{TP}$ & & \\
\hline$M P L$ & p.W515L & & & & & $\mathrm{TP}$ & $\mathrm{TP}$ & & & & & FN & TP \\
\hline NPMI & p.W288fs*12 & & & & & $\mathrm{TP}$ & $\mathrm{TP}$ & & & & & $\mathrm{TP}$ & TP \\
\hline PDGFRA & p.D842V & & & TP & TP & & & & & TP & TP & & \\
\hline \multirow[t]{2}{*}{$S F 3 B 1$} & p.K700E & & & & & $\mathrm{TP}$ & $\mathrm{TP}$ & & & & & $\mathrm{TP}$ & TP \\
\hline & p.K666N & & & & & TP & TP & & & & & $\mathrm{TP}$ & TP \\
\hline SRSF2 & p.P95_R102del & & & & & $\mathrm{TP}$ & TP & & & & & $\mathrm{TP}$ & TP \\
\hline$U 2 A F 1$ & p.S34F & & & & & TP & TP & & & & & $\mathrm{TP}$ & TP \\
\hline
\end{tabular}

Fig. 2 Combined analysis of variants with the DNA Amplicon and Pindel apps to evaluate the performance of the custom 22-gene NGS panel based on two identical but independent NGS runs using reference standards. The MPL W515L variant in the Seraseq Myeloid Mutation Mix (Seraseq) was not detected in one of the replicates, giving the custom NGS panel a sensitivity of $99.2 \%$. One variant was detected in the wild-type reference standard TruQ0, giving the panel a specificity of $96.3 \%$. The custom NGS panel also had a positive predictive value of $97.7 \%$, an average intra-run and inter-run concordance of 98.8\% [range 95.2-100\%] and 99.0\% [range 95.2-100\%] respectively, and was able to detect variants at as low as $1 \%$ allele frequency. FP, False positive; TP, True positive; FN; False negative; Rep 1, Replicate 1; Rep 2, Replicate 2

GGCAGAGGCAGCAGCA) stopgain variant (Sample $04, \mathrm{PV}$ ) was found to be located in the same dbSNP cluster rs750318549 as the previously reported ASXL1 p.Gly646Trpfs"12 (c.1934dupG) stopgain, which is the most common ASXL1 mutation accounting for $>50 \%$ of all identified ASXL1 mutations in myeloid malignancies [19] (Table 2). While ASXL1 p.Gly646Trpfs*12 is the result of a duplication of a $G$ nucleotide within a homopolymer region of eight $G$ nucleotides [19], the variant $A S X L 1$ p.Gly646Trpfs"10 identified in this study is the result of an insertion of 67 nucleotides at position chr20: 31022442, making it a novel stopgain variant. Two other $A S X L 1$ variants identified in this study,
ASXL1 p.Leu731Tyrfs*12 (Sample 09, overt-PMF) and Q1433Q (Sample 03, ET) were also putative novel variants with no dbSNP, COSMIC or ClinVar data; whereas the ASXL1 p.Tyr591*variant (Sample 10, overt-PMF) has been reported in various diseases including ET and MF $[9,20]$, MDS [21], chronic myelomonocytic leukaemia [22], AML [23], mast cell neoplasm [24] and CNL [25], as well as breast cancer [26], but has not been previously reported in PV (Table 2).

Seven TET2 variants were identified in this study, of which, TET2 p.Tyr1245Cys (Sample 08, pre-PMF) was not found to be reported in dbSNP, COSMIC or the ClinVar database. The TET2 p.Ala304Val (Sample 10, 


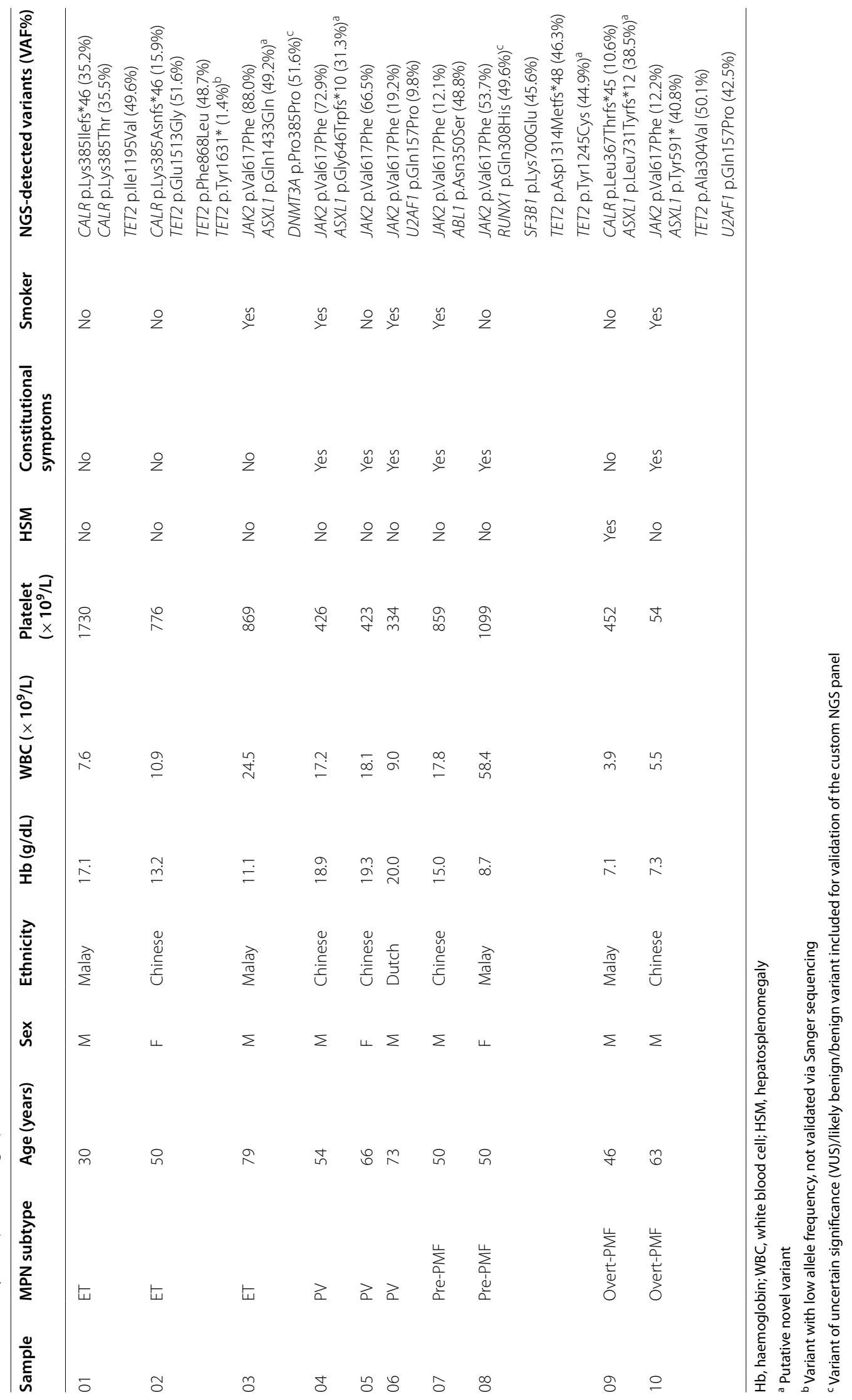




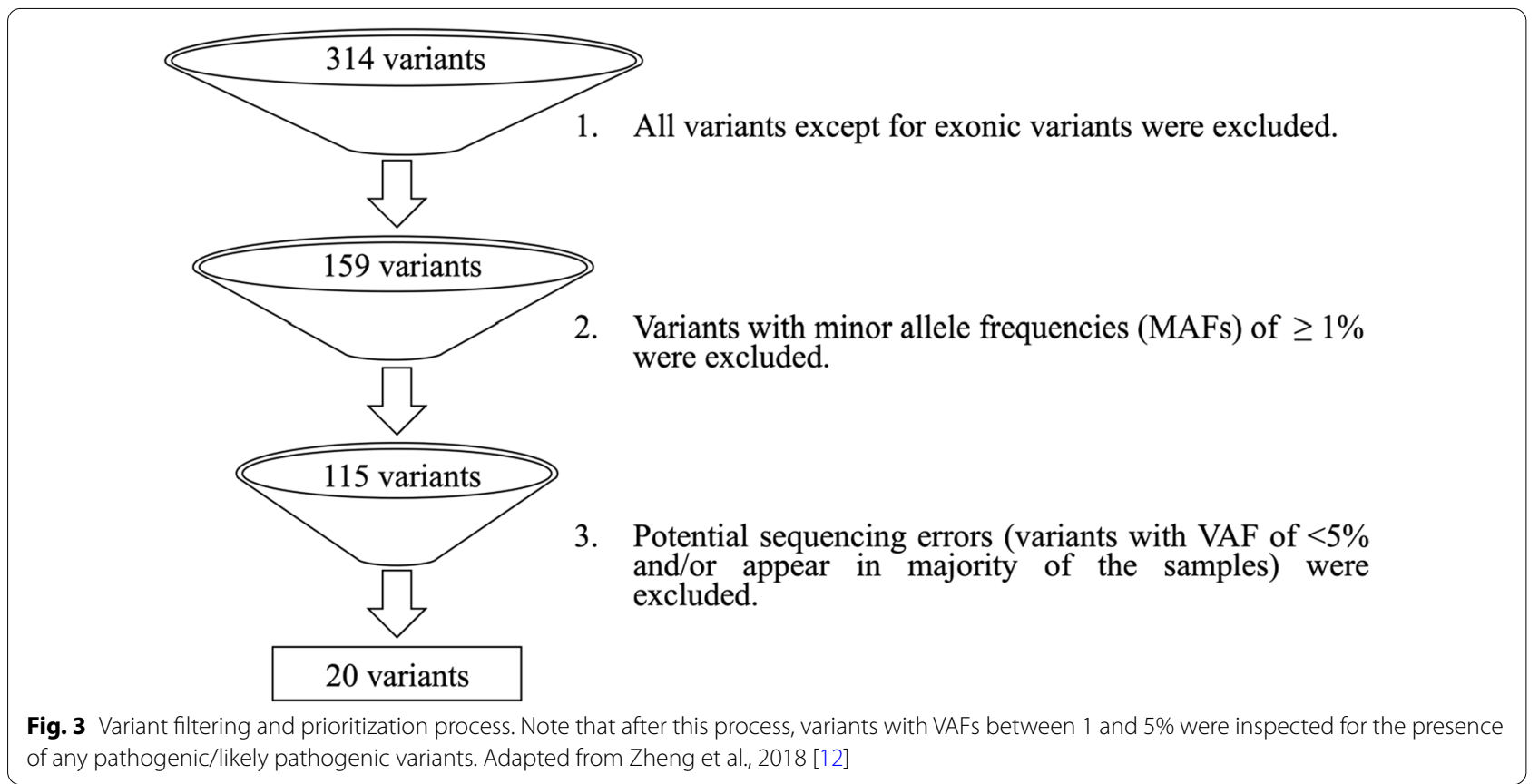

overt-PMF) and p.Phe868Leu (Sample 02, ET) variants have not been previously reported in MPN. TET2 p.Ala304Val has only been previously identified in melanoma [27], while TET2 p.Phe868Leu has been previously identified in estrogen- and progesterone-receptor positive breast cancer [28], adult $\mathrm{T}$ cell lymphoma/leukaemia [29], and MDS [30, 31]. The TET2 p.Asp1314Metfs*48 variant (Sample 08, pre-PMF) has been reported in ET [32] and MDS [21]. Two other variants were reported in dbSNP-TET2 p.Ile1195Val (rs568009712) (Sample 01, ET) and p.Glu1513Gly (rs553669299) (Sample 02, $\mathrm{ET})$, but the associated disease(s) are unknown. Of note, Sample 02 was found to carry another TET2 variant-the p.Tyr 1631* stopgain, reported as pathogenic in the COSMIC database and previously identified in angioimmunoblastic T cell lymphoma [33], CML, and AML [34] at a VAF of $1.4 \%$ (Table 2).

Two of the most common mutations in SF3B1 and $U 2 A F 1$ were identified in this study, namely U2AF1 p.Gln157Pro (rs371246226, COSM211534, COSM1318797) and SF3B1 p.Lys700Glu (rs559063155, COSM84677) [35-37]. Both variants have been reported to be likely pathogenic in the ClinVar database. SF3B1 p.Lys700Glu was found in a pre-PMF sample (Sample 08) which also harboured the TET2 p.Tyr1245Cys and TET2 p.Asp1314Metfs*48 variants alongside the JAK2 V617F driver mutation. The patient was 50 years of age and presented with abnormally high platelet count $\left(1099 \times 10^{9} / \mathrm{L}\right)$, anaemia $(\mathrm{Hb}=8.7 \mathrm{~g} / \mathrm{dL})$, and constitutional symptoms (Table 1). U2AF1 p.Gln157Pro was identified in PV and overt-PMF (Sample 06 and Sample 10 , respectively) (Table 2 ). The overt-PMF sample (Sample 10) also harboured the $A S X L 1$ p.Tyr591* stopgain variant in addition to the $J A K 2 \mathrm{~V} 617 \mathrm{~F}$ driver mutation. The patient was 63 years of age and presented with severe anaemia $(\mathrm{Hb}=7.3 \mathrm{~g} / \mathrm{dL})$, leucopenia $\left(\mathrm{WBC}=5.5 \times 10^{9} / \mathrm{L}\right)$ and thrombocytopenia (Platelet $=54 \times 10^{9} / \mathrm{L}$ ), with constitutional symptoms (Table 1).

\section{Discussion}

In this study, we evaluated the performance of our custom 22-gene NGS panel using reference standards and subsequently, a small cohort of clinical MPN samples. The 22 genes selected in this panel are known to be frequently mutated in myeloid neoplasms (not necessarily MPNs), and are known disease markers with diagnostic, prognostic and/or therapeutic value. First, the performance of the custom NGS panel was technically validated using reference standards in two identical but independent sequencing runs. The combined analysis of variants detected by the DNA Amplicon and Pindel tools revealed that the panel achieved high sensitivity, specificity, concordance and positive predictive values. The overall good performance of the panel was further supported in its ability to detect variants with VAF values as low as 1\% (Fig. 2). Overall, the depth-of-coverage achieved across all amplicons was around our targeted coverage of 5000x (Additional file 8: Fig. S2). However, the low average depth-of-coverage of the MPL amplicon (AMPL117202, MPL exon 10, chr1:43814902-43815103) 
Table 2 Details of all NGS detected variants across the 10 clinical MPN samples

\begin{tabular}{|c|c|c|c|c|c|c|c|c|}
\hline Gene & c.DNA & Aa change & $\begin{array}{l}\text { Conse- } \\
\text { quence }\end{array}$ & dbSNP & $\begin{array}{l}\text { ClinVar assertion/ } \\
\text { COSMIC ID }\end{array}$ & Sample & $\begin{array}{l}\text { VAF } \\
\text { (\%) }\end{array}$ & Sanger \\
\hline CALR & c.1092_1143del & p.Leu367Thrfs*45 & Fs del & NA & $\begin{array}{l}\text { Pathogenic/ } \\
\text { COSM1738055 }\end{array}$ & 09, Overt-PMF & 10.6 & $\checkmark$ \\
\hline CALR & c.1154_1155insTTGTC & p.Lys385Asnfs*46 & Fs ins & rs765476509 & COSM1738056 & $02, \mathrm{ET}$ & 15.9 & $\checkmark$ \\
\hline CALR & c.1153_1154insTATGT & p.Lys385Ilefs*46 & Fs ins & NA & COSM5985669 & 01, ET & 35.2 & $\checkmark$ \\
\hline CALR & C. $1154 A>C$ & p.Lys385Thr & nSNV & rs1024435400 & NA & 01, ET & 35.5 & $\checkmark$ \\
\hline \multirow[t]{7}{*}{ JAK2 } & \multirow[t]{7}{*}{ c.1849G > T } & \multirow[t]{7}{*}{ p.Val617Phe } & \multirow[t]{7}{*}{ nSNV } & \multirow[t]{7}{*}{ rs77375493 } & \multirow[t]{7}{*}{ Pathogenic/COSM12600 } & 07, Pre-PMF & 12.1 & $\times$ \\
\hline & & & & & & 10, Overt-PMF & 12.2 & $x$ \\
\hline & & & & & & $06, P V$ & 19.2 & $\checkmark$ \\
\hline & & & & & & 08, Pre-PMF & 53.7 & $\checkmark$ \\
\hline & & & & & & 05, PV & 66.5 & $\checkmark$ \\
\hline & & & & & & 04, PV & 72.9 & $\checkmark$ \\
\hline & & & & & & 03, ET & 88.0 & $\checkmark$ \\
\hline$A B L 1$ & c. $1049 A>G$ & p.Asn350Ser & nSNV & rs144448357 & NA & 07, Pre-PMF & 48.8 & $\checkmark$ \\
\hline$A S X L 1$ & $\begin{array}{l}\text { c.1927_1928insGGGGGGGGTG } \\
\text { GCCCGGGTGGAGGTGGCGG } \\
\text { CGGGGCCACCGATGAGGGG } \\
\text { GGGGGCAGAGGCAGCAGCA }\end{array}$ & p.Gly646Trpfs*10a & stopgain & rs750318549 & NA & 04, PV & 31.3 & $\checkmark$ \\
\hline$A S X L 1$ & c.1772dupA & p.Tyr591* & stopgain & rs762036456 & $\begin{array}{l}\text { COSM4169775, } \\
\text { COSM4169776 }\end{array}$ & 10, Overt-PMF & 40.8 & $\checkmark$ \\
\hline$A S X L 1$ & c.2190del & p.Leu731Tyrfs* $12^{a}$ & Fs del & NA & NA & 09, Overt-PMF & 38.5 & $\checkmark$ \\
\hline ASXL1 & c. $4299 A>G$ & p.Gln1433G $\ln ^{a}$ & sSNV & NA & NA & 03, ET & 49.2 & $\checkmark$ \\
\hline DNMT3A & c. $1155 G>A$ & p.Pro385Pro ${ }^{c}$ & sSNV & rs368009374 & VUS/likely benign & 03, ET & 51.6 & NA \\
\hline RUNX1 & $c .924 \mathrm{G}>\mathrm{T}$ & p.Gln308His ${ }^{c}$ & nSNV & rs80314254 & Benign & 08, Pre-PMF & 49.6 & $\checkmark$ \\
\hline SF3B1 & c. $2098 \mathrm{~A}>\mathrm{G}$ & p.Lys700Glu & nSNV & rs559063155 & $\begin{array}{l}\text { Likely pathogenic/ } \\
\text { COSM84677 }\end{array}$ & 08, Pre-PMF & 45.6 & $\checkmark$ \\
\hline TET2 & c.911C>T & p.Ala304Val & nSNV & NA & $\begin{array}{l}\text { COSM5610834, } \\
\text { COSM5610835 }\end{array}$ & 10, Overt-PMF & 50.1 & $\checkmark$ \\
\hline TET2 & c. $2604 \mathrm{~T}>\mathrm{G}$ & p.Phe868Leu & nSNV & rs147836249 & COSM87107 & $02, \mathrm{ET}$ & 48.7 & $\checkmark$ \\
\hline TET2 & c. $3583 A>G$ & p.lle1195Val & nSNV & rs568009712 & NA & 01, ET & 49.6 & $\checkmark$ \\
\hline TET2 & c. $3734 A>G$ & p.Tyr1245Cys ${ }^{\mathrm{a}}$ & nSNV & NA & NA & 08, Pre-PMF & 44.9 & $\checkmark$ \\
\hline TET2 & c.3937del & $\begin{array}{l}\text { p.Asp- } \\
\text { 1314Metfs*48 }\end{array}$ & Fs del & NA & COSM4383928 & 08, Pre-PMF & 46.3 & $\checkmark$ \\
\hline TET2 & c. $4893 \mathrm{~T}>\mathrm{A}$ & p.Tyr1631*b & stopgain & NA & COSM97191 & $02, \mathrm{ET}$ & 1.4 & NA \\
\hline TET2 & $c .4538 A>G$ & p.Glu1513Gly & nSNV & rs553669299 & NA & $02, \mathrm{ET}$ & 51.6 & $\checkmark$ \\
\hline U2AF1 & C. $470 A>C$ & p.Gln157Pro & nSNV & rs371246226 & Likely pathogenic/ & 06, PV & 9.8 & $x$ \\
\hline & & & & rs371246226 & $\begin{array}{l}\text { COSM211534, } \\
\text { COSM1318797 }\end{array}$ & 10, Overt-PMF & 42.5 & $\checkmark$ \\
\hline
\end{tabular}

Aa change, amino acid change; Fs del, frameshift deletion; Fs ins, frameshift insertion; $\checkmark$, Sanger detected; $\times$, Sanger undetected; NA, data not available

a Putative novel variant

${ }^{b}$ Variant with low allele frequency, not validated via Sanger sequencing

${ }^{\mathrm{C}}$ Variant of uncertain significance (VUS)/likely benign/benign variant included for validation of the custom NGS panel

at $<100 \times$ could potentially lead to false negative results. In order for the panel to be used in MPN diagnostics, optimisation of the sequencing coverage for the $M P L$ region is critical for the detection of driver mutations in MPL. In addition, AMPL89337 (DNMT3A exon 17, chr2:25464411-25464625), AMPL1156 (TP53 exon 4/exon 5 chr17:7578360-7578579) and AMPL90417 (ASXL1 exon 13, chr20:31022935-31023187) were found to have average depths-of-coverage of $<1000 x$ (Additional file 8: Fig. S2, Additional file 10).

Uneven coverage or coverage bias may originate from various steps in the NGS workflow. The use of the PCR method during NGS library preparation has been found to be the primary contributor [38-40]. In PCR, the annealing efficiency of primers is commonly hindered by templates that are GC-rich that tend to remain double-stranded, as 


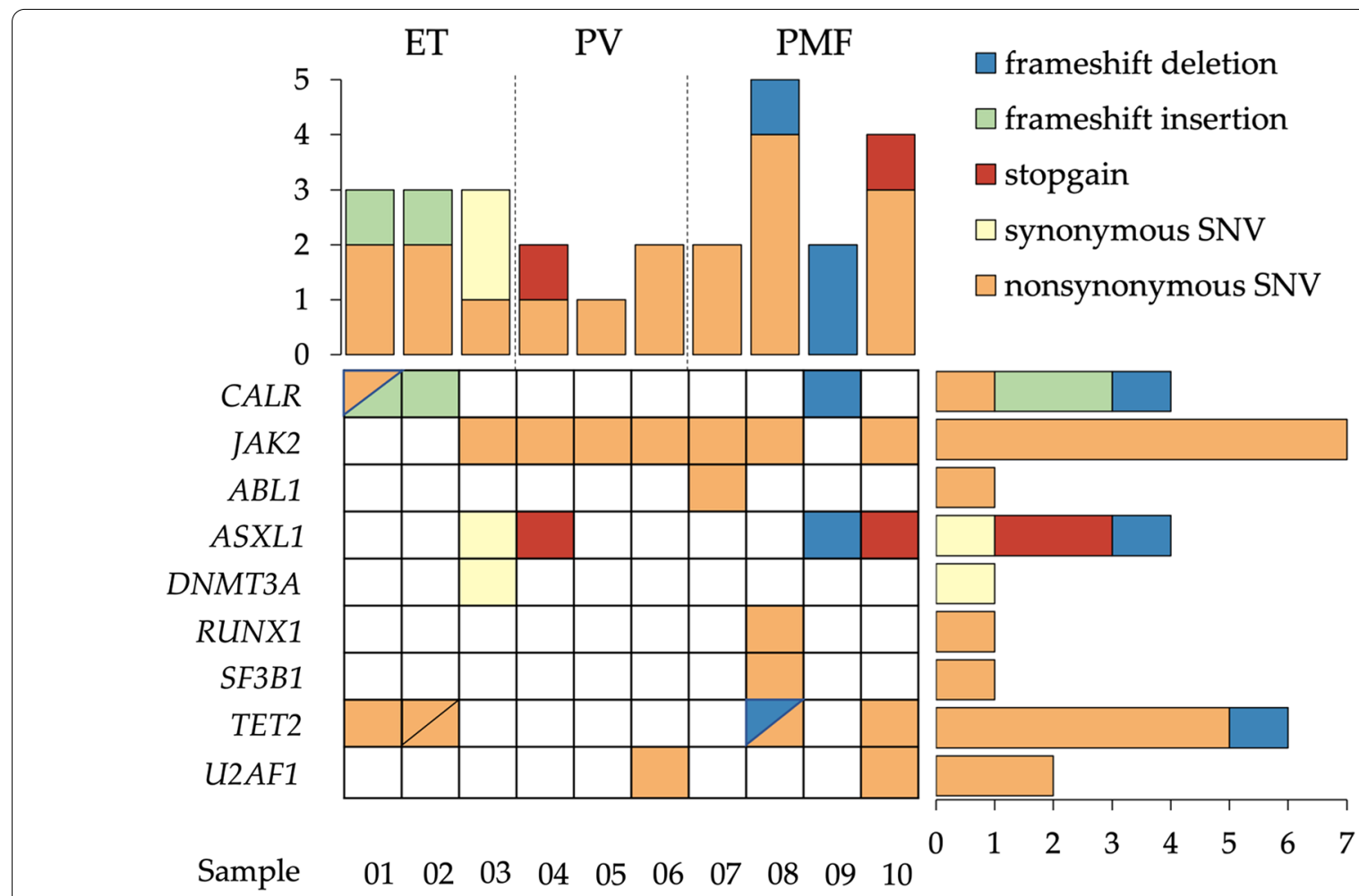

Fig. 4 Variants detected in the clinical MPN samples with VAF $>5 \%$. Note that Sample 02 also carries another variant in TET2 with a VAF of $1.4 \%$ (not shown in Fig. 4)

well as templates that are AT-rich (or GC-poor) that anneal poorly to primers. As a result, GC-and AT-rich regions (also known as low-complexity regions) are often poorly amplified and manifest in NGS as regions with low sequencing coverage. Further analysis of AMPL117202 showed that it had a GC content of $61 \%$ and AT content of $39 \%$, and a plot of the nucleotide distribution revealed that the GC content was $>60 \%$ in the majority of 30 bp windows (Additional file 11: Fig. S4). Hence, it is likely that the majority of the AMPL117202 amplicons remained annealed as doublestranded DNA during PCR, leading to inefficient amplification and subsequent low NGS coverage of the amplicons. Future studies should involve the review of targeted regions and further optimisation of the NGS library preparation to improve the coverage of $\mathrm{GC}$-rich regions, i.e. optimising PCR amplification conditions by using lower primer-extension temperature [38]. In order to rule out false negatives due to coverage bias, variants located in DNMT3A exon 17 and TP53 exon 4/exon 5 should also be used to validate the performance of the custom panel.

From the screening of clinical MPN samples with the custom NGS panel, 21 unique variants including MPN driver mutations in $J A K 2$ and $C A L R$, and one pathogenic stopgain variant in TET2 with a VAF of $1.4 \%$ were identified. The ASXL1 p.Leu731Tyrfs*12, p.Gln1433Gln, p.Gly646Trpfs*10 and TET2 p.Tyr1245Cys variants were identified as putative novel variants, whereas reported and likely pathogenic variants were identified in $A B L 1, S F 3 B 1$ and $U 2 A F 1$. The study findings support the notion that the co-presence of multiple variants within a single sample results in a potentially synergistic effect that promotes disease development and progression, and contributes towards higher symptom burden, poorer prognosis, higher risk of leukaemic transformation, and drug resistance [21, 41-46]. In $A B L 1$, point mutations that confer resistance against tyrosine kinase inhibitors in patients with CML have been discovered in more than 50 different hotspots in the kinase domain [47-50]. It is possible that $A B L 1$ p.Asn350Ser identified in this study may confer drug resistance in a similar fashion to those that have previously been identified.

As MPNs are a clonally heterogenous and multifactorial group of diseases, differences in gene dosage and clonal architecture, the order of mutation acquisition and even germline predisposition can contribute towards MPN pathogenesis as well as differences in disease phenotype and prognosis $[51,52]$. In addition, other factors such as lineage bias in haematopoietic stem cells, changes in the bone marrow microenvironment, and aging have also been reported [53]. Further studies should aim to experimentally characterise the functional impact of the variants identified and 
investigate their possible synergistic effects and biological significance in myeloid disorders, especially in MPN. The putative novel variants should be further investigated using in vitro candidate gene approaches via protein function assays and bioinformatics analyses in order to elucidate their impact especially in poorly characterized proteins $[54,55]$.

In this study, all NGS-identified variants were confirmed via bidirectional Sanger sequencing except for TET2 p.Tyr1631*, JAK2 V617F and $U 2 A F 1$ p.Gln157Pro where the allelic frequencies were $<15 \%$, whereas DMNT3A p.Pro385Pro was not confirmed due to nonspecific primer binding during the PCR step (Table 2, Additional file 9: Fig. S3). Sanger sequencing is widely regarded as the 'gold standard' for the validation of NGS-detected variants as it can discriminate true variants from NGS artifacts or sequencing errors [56]. However, as the Sanger method relies on the detection of fluorescence, it has limited sensitivity when detecting variants with low allele frequencies; particularly in mosaic tumour samples, leading to false negative results [5759]. The sensitivity of Sanger sequencing has been reported to be around $15-20 \%$ allele frequency, whereas NGS has a sensitivity of approximately $1 \%$ allele frequency $[59,60]$. Therefore, best practice standards for NGS variant confirmation should include alternative methods with higher sensitivity, such as droplet digital PCR or allele-specific qPCR, followed by high-resolution melting curve analysis for the confirmation of variants with low allele frequencies $[56,60$, 61]. Nevertheless, such methods are also accompanied by technical limitations and caveats which should be addressed and optimised for their specific intended purposes [62].

Future studies may also benefit from the use of an expanded bioinformatics pipeline which will ultimately provide a greater wealth of information to the clinician, such as the indication of variant germline/somatic status, the identification of clonal haematopoiesis of indeterminate potential (CHIP, which shows evidence of clonal expansion), monitoring of minimal residual disease, determination of disease predisposition, as well as accurate prediction of treatment response/outcome [63, 64]. A larger study cohort as well as the collection of samples and clinical data at follow-up as well as data on response to therapy and survival will allow for better genotype-phenotype associations and contribute towards the better understanding of MPNs.

\section{Conclusions}

In summary, the custom NGS panel enabled the detection of known MPN-associated genetic variants, as well as the identification of novel variants of potential biological significance, indicating its potential clinical utility in the genetic profiling of MPN patients. However, further performance optimisation is required especially for regions with poor coverage depth to ensure that the custom NGS panel will serve as a robust and reliable tool for the personalised management of MPNs. It is hoped that the data generated from the screening of MPN patients using the custom NGS panel will also contribute towards the MPN knowledgebase, and support the adoption of more accurate genomics-based disease classification as well as prognostic frameworks for the management of MPNs.

\begin{abstract}
Abbreviations
AML: Acute myeloid leukaemia; bp: Base pair; CHIP: Clonal haematopoiesis of indeterminate potential; CML: Chronic myeloid leukaemia; CNL: Chronic neutrophilic leukaemia; COSMIC: Catalogue of somatic mutations in cancer; DNA: Deoxyribonucleic acid; dNTP: Dideoxynucleotide phosphates; ET: Essential thrombocythaemia; ExAC: Exome Aggregation Consortium; ESP6500: NHLBI Exome Sequencing Project; gnomAD: Genome Aggregation Database; IGV: Integrative genomics viewer; MDS: Myelodysplastic syndrome; MDS/MPN: Myelodysplastic/myeloproliferative neoplasms; MF: Myelofibrosis; MAF: Minor allele frequency; MPN: Myeloproliferative neoplasms; NGS: Next-generation sequencing; nSNV: Nonsynonymous single nucleotide variant; Ph: Philadelphia; PMF: Primary myelofibrosis; PV: Polycythaemia vera; SNV: Single nucleotide variant; SSNV: Synonymous single nucleotide variant; TN: Triple-negative; VAF: Variant allele frequency.
\end{abstract}

\section{Supplementary Information}

The online version contains supplementary material available at https://doi. org/10.1186/s12920-021-01145-0.

Additional file 1. Table S1. Targeted exons of $22 \mathrm{MPN}$-associated genes in the custom NGS panel.

Additional file 2. Table S2. Amplicon regions.

Additional file 3. Table S3. Details of variants present in reference standards.

Additional file 4. Table S4. Primers used in variant confirmation.

Additional file 5. Fig. S1. Representative image of (a) Labchip gel and (b) multiple overlay electropherogram of the constructed libraries of the reference standards. The image shows the quality and quantity of input DNA for the second NGS run for the technical validation of the custom NGS panel. The majority of the constructed libraries were within the targeted size range of $400 \mathrm{bp}$

Additional file 6. Table S5. Sequencing metrics of NGS runs for the technical validation of the custom NGS panel.

Additional file 7. Table S6. Amplicon coverage data for the technical validation of the custom NGS panel.

Additional file 8. Fig. S2. Amplicon coverage across reference standards in the technical validation of the custom NGS panel. More details on the amplicon regions can be found in Additional file 2: Table S2 and Additional file 10.

Additional file 9. Fig. S3. Gel electrophoresis images

Additional file 10. Amplicon coverage. Sheet 1 shows the genomic sequences covered by each amplicon, whereas Sheet 2 shows the depthof-coverage per amplicon for each of the samples across the two NGS runs.

Additional file 11. Fig. S4. Distribution of GC content across AMPL117202 (MPL exon 10, chr1:43814902-43815103).

\section{Acknowledgements}

The authors would like to thank Sunway Medical Centre for funding the study.

\section{Authors' contributions}

Conceptualization, CLW and AV; methodology, CLW, AV, YPC, and NZA; software, YPC and YMP; validation, YPC, NZA and JT; formal analysis, JT and 
YPC; investigation, JT, YPC and NZA; resources, CLW, AV, KMC, VS and YMP; data curation, JT, YPC; writing—original draft preparation, JT; writing—review and editing, JT, YPC, NZA, CKM, VS, NRT, YMP, AV, MAL, and CLW; visualization, JT and YPC; supervision, YPC, NZA, CLW, AV and MAL; project administration, YPC, NZA and JT; funding acquisition, CLW. All authors read and approved the final manuscript.

\section{Funding}

This research was funded by the Sunway Medical Centre Research Fund, Grant Number SRC/F/18/006. The APC was funded by the Sunway Medical Centre Publication Fund.

\section{Availability of data and materials}

The datasets generated and/or analysed during the current study are available in the NCBI BioProject repository, PRJNA754686.

\section{Declarations}

\section{Ethics approval and consent to participate}

The study was conducted in accordance to the guidelines of the Declaration of Helsinki, and as approved by the Sunway Medical Centre Independent Research Ethics Committee (SREC 008/2018/FR, date of approval 27th June 2018), Universiti Kebangsaan Malaysia Medical Centre Research Ethics Committee (UKM FPR.4/244/FF-2018-420, date of approval 29th November 2018), Medical Research and Ethics Committee Ministry of Health Malaysia (MREC 8KKM/NIHSEC/P-19-99, date of approval 22nd January 2019), and Sunway University Research Ethics Committee (PGSUREC 2019/010, date of approval 9th April 2019). Informed consent was obtained from all subjects involved in the study.

\section{Consent for publication}

Informed consent was obtained from the funding institution and all subjects involved in the study.

\section{Competing interests}

The authors declare that they have no competing interests.

\section{Author details}

${ }^{1}$ Department of Biological Sciences, School of Medical and Life Sciences, Sunway University, Petaling Jaya, Selangor, Malaysia. ${ }^{2}$ Clinical Research Centre, Sunway Medical Centre, Petaling Jaya, Selangor Darul Ehsan, Malaysia. ${ }^{3}$ Molecular Diagnostics Laboratory, Sunway Medical Centre, Petaling Jaya, Selangor Darul Ehsan, Malaysia. ${ }^{4}$ Haematology Unit, Department of Medicine, Sunway Medical Centre, Petaling Jaya, Selangor Darul Ehsan, Malaysia. ${ }^{5}$ Haematology Department, Ampang Hospital, Kuala Lumpur, Malaysia. ${ }^{6}$ Haematology Unit, Department of Medicine, Universiti Kebangsaan Malaysia Medical Centre, Kuala Lumpur, Malaysia. ${ }^{7}$ School of Data Sciences, Perdana University, Serdang, Selangor, Malaysia. ${ }^{8}$ Centre for Haematology, Hammersmith Hospital, London, UK. ${ }^{9}$ Faculty of Medicine, Imperial College London, London, UK.

Received: 15 July 2021 Accepted: 6 December 2021 Published online: 15 January 2022

\section{References}

1. Cervantes F, Passamonti F, Barosi G. Life expectancy and prognostic factors in the classic BCR/ABL-negative myeloproliferative disorders. Leukemia. 2008;22(5):905-14.

2. Vannucchi AM, Barbui T, Cervantes F, Harrison C, Kiladjian JJ, Kroger N, et al. Philadelphia chromosome-negative chronic myeloproliferative neoplasms: ESMO Clinical Practice Guidelines for diagnosis, treatment and follow-up. Ann Oncol Off J Eur Soc Med Oncol. 2015;26(Suppl 5):v85-99.

3. Mullally A, Bruedigam C, Poveromo L, Heidel FH, Purdon A, Vu T, et al. Depletion of Jak2V617F myeloproliferative neoplasm-propagating stem cells by interferon-a in a murine model of polycythemia vera. Blood. 2013;121(18):3692-702.

4. Swerdlow SH, Campo E, Harris NL, Jaffe E, Pileri S, Stein H, et al. WHO classification of tumours of haematopoietic and lymphoid tissues. 4th ed. Lyon: International Agency for Research on Cancer (IARC) Press; 2017.
5. Arber DA, Orazi A, Hasserjian R, Thiele J, Borowitz MJ, Le Beau MM, et al. The 2016 revision to the World Health Organization classification of myeloid neoplasms and acute leukemia. Blood. 2016;127(20):2391-405.

6. Buhr T, Hebeda K, Kaloutsi V, Porwit A, Van der Walt J, Kreipe H. European Bone Marrow Working Group trial on reproducibility of World Health Organization criteria to discriminate essential thrombocythemia from prefibrotic primary myelofibrosis. Haematologica. 2012;97(3):360-5.

7. Wilkins BS, Erber WN, Bareford D, Buck G, Wheatley K, East CL, et al. Bone marrow pathology in essential thrombocythemia: interobserver reliability and utility for identifying disease subtypes. Blood. 2008;111(1):60-70.

8. Brousseau M, Parot-Schinkel E, Moles MP, Boyer F, Hunault M, Rousselet MC. Practical application and clinical impact of the WHO histopathological criteria on bone marrow biopsy for the diagnosis of essential thrombocythemia versus prefibrotic primary myelofibrosis. Histopathology. 2010;56(6):758-67.

9. Grinfeld J, Nangalia J, Baxter EJ, Wedge DC, Angelopoulos N, Cantrill R, et al. Classification and personalized prognosis in myeloproliferative neoplasms. N Engl J Med. 2018;379(15):1416-30.

10. Ye K, Schulz MH, Long Q, Apweiler R, Ning Z. Pindel: a pattern growth approach to detect break points of large deletions and medium sized insertions from paired-end short reads. Bioinformatics. 2009;25(21):2865-71.

11. Dai Y, Liang S, Dong X, Zhao Y, Ren H, Guan Y, et al. Whole exome sequencing identified a novel DAG1 mutation in a patient with rare, mild and late age of onset muscular dystrophy-dystroglycanopathy. J Cell Mol Med. 2019;23(2):811-8.

12. Zheng $Y, X u$ J, Liang S, Lin D, Banerjee S. Whole exome sequencing identified a novel heterozygous mutation in HMBS gene in a Chinese patient with acute intermittent porphyria with rare type of mild anemia. Front Genet. 2018;9:129.

13. Sherry ST, Ward MH, Kholodov M, Baker J, Phan L, Smigielski EM, et al. dbSNP: the NCBI database of genetic variation. Nucleic Acids Res. 2001;29(1):308-11.

14. Landrum MJ, Lee JM, Riley GR, Jang W, Rubinstein WS, Church DM, et al. ClinVar: public archive of relationships among sequence variation and human phenotype. Nucleic Acids Res. 2014;42(Database issue):D980-5.

15. Tate JG, Bamford S, Jubb HC, Sondka Z, Beare DM, Bindal N, et al. COSMIC: the catalogue of somatic mutations in cancer. Nucleic Acids Res. 2018;47(D1):D941-7.

16. Yates AD, Achuthan P, Akanni W, Allen J, Allen J, Alvarez-Jarreta J, et al. Ensembl 2020. Nucleic Acids Res. 2019;48(D1):D682-8.

17. Ye J, Coulouris G, Zaretskaya I, Cutcutache I, Rozen S, Madden TL. PrimerBLAST: a tool to design target-specific primers for polymerase chain reaction. BMC Bioinform. 2012;13:134.

18. Waterhouse AM, Procter JB, Martin DMA, Clamp M, Barton GJ. Jalview Version 2-a multiple sequence alignment editor and analysis workbench. Bioinformatics. 2009;25(9):1189-91.

19. Abdel-Wahab O, Kilpivaara O, Patel J, Busque L, Levine RL. The most commonly reported variant in ASXL1 (c.1934dupG;p.Gly646TrpfsX12) is not a somatic alteration. Leukemia. 2010;24(9):1656-7.

20. Nangalia J, Massie CE, Baxter EJ, Nice FL, Gundem G, Wedge DC, et al. Somatic CALR mutations in myeloproliferative neoplasms with nonmutated JAK2. N Engl J Med. 2013:369(25):2391-405.

21. Papaemmanuil E, Gerstung M, Malcovati L, Tauro S, Gundem G, Van Loo P, et al. Clinical and biological implications of driver mutations in myelodysplastic syndromes. Blood. 2013;122(22):3616-27.

22. Grossmann V, Kohlmann A, Eder C, Haferlach C, Kern W, Cross NC, et al. Molecular profiling of chronic myelomonocytic leukemia reveals diverse mutations in $>80 \%$ of patients with TET2 and EZH2 being of high prognostic relevance. Leukemia. 2011;25(5):877-9.

23. Pratcorona M, Abbas S, Sanders MA, Koenders JE, Kavelaars FG, Erpelinck-Verschueren CA, et al. Acquired mutations in ASXL1 in acute myeloid leukemia: prevalence and prognostic value. Haematologica. 2012;97(3):388-92.

24. Traina F, Visconte $V$, Jankowska AM, Makishima $H$, O'Keefe $C L$, Elson $P$, et al. Single nucleotide polymorphism array lesions, TET2, DNMT3A, ASXL1 and CBL mutations are present in systemic mastocytosis. PLOS ONE. 2012;7(8):e43090.

25. Cui Y, Li B, Jiang Q, Xu Z, Qin T, Zhang P, et al. CSF3R, ASXL1, SETBP1, JAK2 V617F and CALR mutations in chronic neutrophilic leukemia. Zhonghua xue ye xue za zhi = Zhonghua xueyexue zazhi. 2014;35(12):1069-73. 
26. Ross JS, Gay LM, Wang K, Ali SM, Chumsri S, Elvin JA, et al. Nonamplification ERBB2 genomic alterations in 5605 cases of recurrent and metastatic breast cancer: an emerging opportunity for anti-HER2 targeted therapies. Cancer. 2016;122(17):2654-62

27. Shain AH, Garrido M, Botton T, Talevich E, Yeh I, Sanborn JZ, et al. Exome sequencing of desmoplastic melanoma identifies recurrent NFKBIE promoter mutations and diverse activating mutations in the MAPK pathway. Nat Genet. 2015;47(10):1194-9.

28. Yap YS, Singh AP, Lim JHC, Ahn JH, Jung KH, Kim J, et al. Elucidating therapeutic molecular targets in premenopausal Asian women with recurrent breast cancers. NPJ Breast Cancer. 2018;4:19.

29. Shimoda K, Shide K, Kameda T, Hidaka T, Kubuki Y, Kamiunten A, et al. TET2 mutation in adult T-Cell leukemia/lymphoma. J Clin Exp Hematopathol. 2015;55(3):145-9.

30. Wang J, Ai X, Gale RP, Xu Z, Qin T, Fang L, et al. TET2, ASXL1 and EZH2 mutations in Chinese with myelodysplastic syndromes. Leuk Res. 2013;37(3):305-11.

31. Metzeler KH, Maharry K, Radmacher MD, Mrózek K, Margeson D, Becker $\mathrm{H}$, et al. TET2 mutations improve the new European LeukemiaNet risk classification of acute myeloid leukemia: a Cancer and Leukemia Group B study. J Oncol Off J Am Soc Clin Oncol. 2011;29(10):1373-81.

32. Ha JS, Jeon DS, Kim JR, Ryoo NH, Suh JS. Analysis of the ten-eleven translocation 2 (TET2) gene mutation in myeloproliferative neoplasms. Ann Clin Lab Sci. 2014;44(2):173-9.

33. Odejide O, Weigert O, Lane AA, Toscano D, Lunning MA, Kopp N, et al. A targeted mutational landscape of angioimmunoblastic T-cell lymphoma. Blood. 2014;123(9):1293-6.

34. Roche-Lestienne C, Marceau A, Labis E, Nibourel O, Coiteux V, Guilhot J, et al. Mutation analysis of TET2, IDH1, IDH2 and ASXL1 in chronic myeloid leukemia. Leukemia. 2011;25(10):1661-4.

35. Graubert TA, Shen D, Ding L, Okeyo-Owuor T, Lunn CL, Shao J, et al. Recurrent mutations in the U2AF1 splicing factor in myelodysplastic syndromes. Nat Genet. 2011:44(1):53-7.

36. Yoshida K, Sanada M, Shiraishi Y, Nowak D, Nagata Y, Yamamoto R, et al. Frequent pathway mutations of splicing machinery in myelodysplasia. Nature. 2011:478(7367):64-9.

37. Papaemmanuil E, Cazzola M, Boultwood J, Malcovati L, Vyas P, Bowen D, et al. Somatic SF3B1 mutation in myelodysplasia with ring sideroblasts. N Engl J Med. 2011;365(15):1384-95.

38. Aird D, Ross MG, Chen W-S, Danielsson M, Fennell T, Russ C, et al. Analyzing and minimizing PCR amplification bias in Illumina sequencing libraries. Genome Biol. 2011;12(2):R18.

39. Benjamini Y, Speed TP. Summarizing and correcting the GC content bias in high-throughput sequencing. Nucleic Acids Res. 2012:40(10):e72

40. Pereira R, Oliveira J, Sousa M. Bioinformatics and computational tools for next-generation sequencing analysis in clinical genetics. J Clin Med. 2020;9(1):132.

41. Haferlach T, Nagata Y, Grossmann V, Okuno Y, Bacher U, Nagae G, et al. Landscape of genetic lesions in 944 patients with myelodysplastic syndromes. Leukemia. 2014;28(2):241-7.

42. Thol F, Kade S, Schlarmann C, Löffeld P, Morgan M, Krauter J, et al. Frequency and prognostic impact of mutations in SRSF2, U2AF1, and ZRSR2 in patients with myelodysplastic syndromes. Blood. 2012;119(15):3578-84.

43. Lindsley RC, Saber W, Mar BG, Redd R, Wang T, Haagenson MD, et al. Prognostic mutations in myelodysplastic syndrome after stem-cell transplantation. N Engl J Med. 2017;376(6):536-47.

44. Alberti MO, Srivatsan SN, Shao J, McNulty SN, Chang GS, Miller CA, et al. Discriminating a common somatic ASXL1 mutation (c.1934dup; p.G646Wfs*12) from artifact in myeloid malignancies using NGS. Leukemia. 2018;32(8):1874-8.

45. Grinfeld J, Nangalia J, Green AR. Molecular determinants of pathogenesis and clinical phenotype in myeloproliferative neoplasms. Haematologica. 2017;102(1):7-17.

46. Papaemmanuil E, Gerstung M, Bullinger L, Gaidzik VI, Paschka P, Roberts ND, et al. Genomic classification and prognosis in acute myeloid leukemia. N Engl J Med. 2016;374(23):2209-21.

47. Chandrasekhar C, Kumar PS, Sarma PVGK. Novel mutations in the kinase domain of BCR-ABL gene causing imatinib resistance in chronic myeloid leukemia patients. Sci Rep. 2019;9(1):2412.
48. Soverini S, Rosti G, lacobucci I, Baccarani M, Martinelli G. Choosing the best second-line tyrosine kinase inhibitor in imatinib-resistant chronic myeloid leukemia patients harboring Bcr-Abl kinase domain mutations: how reliable is the $I_{50}$ ? Oncologist. 2011;16(6):868-76.

49. Wylie AA, Schoepfer J, Jahnke W, Cowan-Jacob SW, Loo A, Furet P, et al. The allosteric inhibitor ABL001 enables dual targeting of BCR-ABL1. Nature. 2017;543(7647):733-7.

50. Klco JM, Vij R, Kreisel FH, Hassan A, Frater JL. Molecular pathology of myeloproliferative neoplasms. Am J Clin Pathol. 2010;133(4):602-15.

51. Ortmann CA, Kent DG, Nangalia J, Silber Y, Wedge DC, Grinfeld J, et al. Effect of mutation order on myeloproliferative neoplasms. N Engl J Med. 2015:372(7):601-12

52. Nangalia J, Nice FL, Wedge DC, Godfrey AL, Grinfeld J, Thakker C, et al. DNMT3A mutations occur early or late in patients with myeloproliferative neoplasms and mutation order influences phenotype. Haematologica. 2015;100(11):e438-42.

53. Tan J, Chow YP, Abidin NZ, Veerakumarasivam A, Wong CL. From driver mutations to genomic classification: current \& future perspectives on myeloproliferative neoplasms. Malays J Med Health Sci. 2021;17(1):170-83.

54. Robevska G, van den Bergen JA, Ohnesorg T, Eggers S, Hanna C, Hersmus $R$, et al. Functional characterization of novel NR5A1 variants reveals multiple complex roles in disorders of sex development. Hum Mutat. 2018:39(1):124-39.

55. Wheway G, Nazlamova L, Meshad N, Hunt S, Jackson N, Churchill A. A combined in silico, in vitro and clinical approach to characterize nove pathogenic missense variants in PRPF31 in retinitis pigmentosa. Front Genet. 2019;10:248

56. Beck TF, Mullikin JC, Biesecker LG. Systematic evaluation of sanger validation of next-generation sequencing variants. Clin Chem. 2016;62(4):647-54.

57. Alghasham N, Alnouri Y, Abalkhail H, Khalil S. Detection of mutations in JAK2 exons 12-15 by Sanger sequencing. Int J Lab Hematol. 2016;38(1):34-41.

58. Braunholz D, Obieglo C, Parenti I, Pozojevic J, Eckhold J, Reiz B, et al. Hidden mutations in Cornelia de Lange syndrome limitations of sanger sequencing in molecular diagnostics. Hum Mutat. 2015;36(1):26-9.

59. Paparini A, Gofton A, Yang R, White N, Bunce M, Ryan UM. Comparison of sanger and next generation sequencing performance for genotyping cryptosporidium isolates at the $18 \mathrm{~S}$ rRNA and actin loci. Exp Parasitol. 2015; 151-152:21-7

60. Tsiatis AC, Norris-Kirby A, Rich RG, Hafez MJ, Gocke CD, Eshleman JR, et al Comparison of Sanger sequencing, pyrosequencing, and melting curve analysis for the detection of KRAS mutations: diagnostic and clinical implications. J Mol Diagn. 2010;12(4):425-32.

61. Kuo FC, Mar BG, Lindsley RC, Lindeman NI. The relative utilities of genome-wide, gene panel, and individual gene sequencing in clinical practice. Blood. 2017;130(4):433-9.

62. Słomka M, Sobalska-Kwapis M, Wachulec M, Bartosz G, Strapagiel D. High resolution melting (HRM) for high-throughput genotyping-limitations and caveats in practical case studies. Int J Mol Sci. 2017;18(11):2316.

63. Teer JK, Zhang Y, Chen L, Welsh EA, Cress WD, Eschrich SA, et al. Evaluating somatic tumor mutation detection without matched normal samples. Hum Genom. 2017;11(1):22.

64. Baer C, Walter W, Hutter S, Twardziok S, Meggendorfer M, Kern W, et al. "Somatic" and "pathogenic": is the classification strategy applicable in times of large-scale sequencing? Haematologica. 2019;104(8):1515-20.

\section{Publisher's Note}

Springer Nature remains neutral with regard to jurisdictional claims in published maps and institutional affiliations. 